\title{
Mixed mesodermal sarcoma of the uterus presenting as spontaneous rupture of the kidney
}

\author{
V. COPE* \\ D.M.R.D., F.F.R.
}

D. M. ESSENHIGH

M.Chir., F.R.C.S.

The Radcliffe Infirmary, Oxford

RUPTURE of the kidney or renal pelvis in the absence of trauma is a rare event, and occurs usually in a grossly diseased organ. The subject has been reviewed by Shaw (1957) who found that obstructive hydronephrosis is the commonest condition leading to spontaneous rupture, and this accounted for twenty-nine of forty cases described in the literature.

Our case differs from those described by Shaw in that the obstructing lesion was at the lower end of the ureter and was later shown to be due to a rare tumour, the mixed mesodermal sarcoma of the uterus.

\section{Case report}

Mrs L.P., aged 59, was admitted as an emergency in November 1964 with a 3 week history of colicky pain in the left loin. She had no frequency, dysuria or haematuria. Her appetite was good, bowels were open normally, and she had not noticed any vaginal bleeding or discharge.

On examination, she had a pyrexia of $100^{\circ} \mathrm{F}$ and apart from some tenderness in the left loin, no abnormality could be found. Rectal examination was normal. A clinical diagnosis of pyelonephritis was made, and she was treated with tetracycline $250 \mathrm{mg}$ q.d.s.

Investigations: $\mathrm{Hb} \quad 11.3 \mathrm{~g} / 100 \mathrm{ml}$, WBC $10,300 / \mathrm{mm}^{3}$, ESR 112 , urine culture, light growth of $E$. coli, no acid-fast bacilli seen. Blood urea $24 \mathrm{mg} / 100 \mathrm{ml}$, chest X-ray normal.

An intravenous pyelogram 4 days after admission showed distortion and dilatation of the pelvicalyceal system of the left kidney, with extravasation of contrast medium below the lower pole (Fig. 1). No obvious cause for the extravasation of

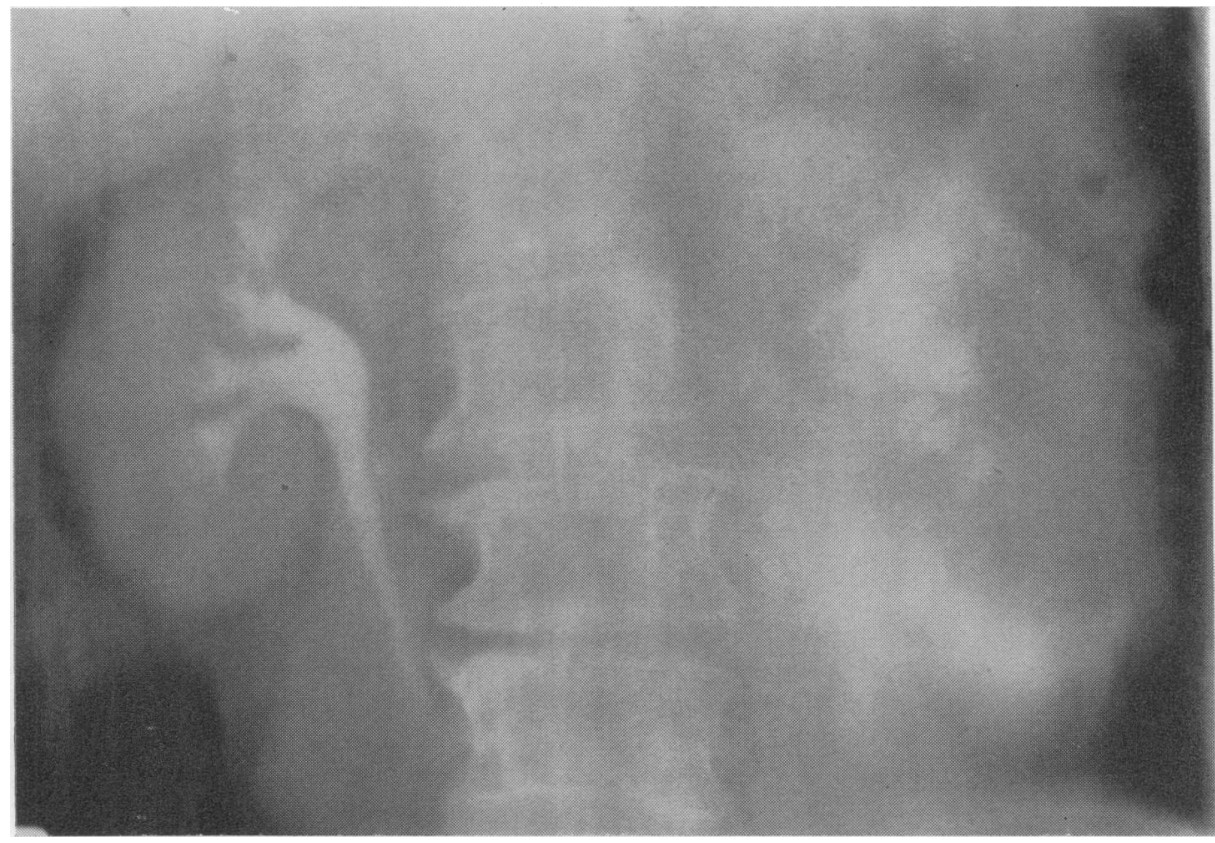

FIG. 1. Tomogram of left kidney at pyelography showing distortion and dilatation of calyceal system with extravasation of contrast medium below the lower pole.

*Present address: Manchester Royal Infirmary. 


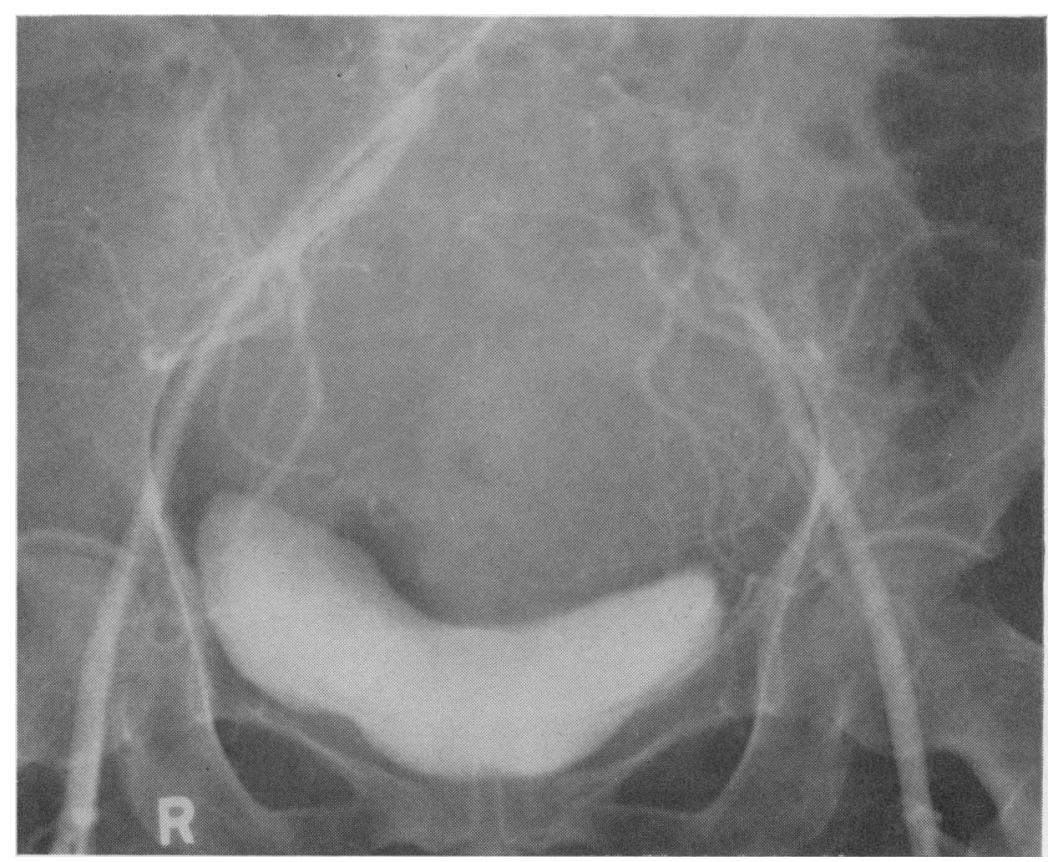

Fig. 2. Arteriogram of pelvic mass found on re-admission. There are no features diagnostic of malignancy.

contrast medium could be seen and a renal arteriogram was performed to exclude the possibility of malignant disease. This showed no evidence of renal tumour. It was thought that the diagnosis was one of spontaneous rupture of the left renal pelvis, with pyelitis, and that conservative treatment should be continued. After 2 weeks there was considerable improvement and she was discharged on tetracycline with arrangements for her readmission after a month with a view to further radiological studies of the renal tract.

A recurrence of more severe pain necessitated readmission as an emergency early in January 1965. There were now signs of peritonitis in the left side of the abdomen which were thought at first to be due to a perinephric abscess. Shortly after this admission she had some vaginal bleeding, and examination then revealed a pelvic mass at first thought clinically to be fibroids. A further arteriogram showed diminished function in the left kidney with no radiological evidence of a perinephric abscess. A pelvic arteriogram confirmed the presence of a pelvic mass, apparently uterine in origin, with no evidence of malignant circulation (Fig. 2). During the next few days, the patient became progressively more ill and the signs of peritonitis gradually increased with a swinging pyrexia indicating abscess formation, necessitating laparotomy.
At operation she was found to have peritonitiš due to a ruptured pericolic abscess in the left iliac fossa, this was apparently caused by a perforated neoplasm of the sigmoid colon. There was extensive malignant infiltration of the pelvis and the uterus was noted to contain a large mass, but it seemed to be inopportune to perform a hysterectomy. The left kidney felt normal on palpation and at this time there was no evidence of peri-renal infection. Local resection of the sigmoid colon with terminal colostomy was performed. Histology of the excised specimen was reported to be a primary leiomyosarcoma of the colon, although the pathologist considered that invasion of the colon from adjacent structures could not be completely excluded. Following operation the patient's condition steadily deteriorated, she developed renal failure, and died 3 weeks later.

Necropsy. The whole of the peritoneal cavity was a matted mass of inflammatory and neoplastic tissue with metastases in the liver. The pelvis was a solid mass of malignant tissue, involving both ueters, and no definite diagnosis could be made on naked-eye examination, although fungating areas within the uterine mass suggested that this was probably malignant as well. There was now bilateral pyelonephritis, and on the left a large pyonephrosis communicated with a perinephric 
abscess. The kidney was not involved by the malignant process. Histology later revealed that the uterine mass was a mixed mesodermal sarcoma with a pattern of fibrosarcoma, chondrosarcoma, and in places osteogenic sarcoma. Review of the segment of colon removed at operation showed that this was in fact invaded from without by secondaries from the primary uterine tumour.

\section{Discussion}

Spontaneous rupture of the renal pelvis is rare and is most commonly caused by a stone impacted at the pelvi-ureteric junction (Mathé, 1932). Cases have been reported of rupture due to secondary deposits in the ureter (Ferguson, 1944), and to pregnancy (Jeppeson, 1961). Pyelonephritis may also lead to spontaneous rupture (Mathé, 1932; Jeppeson, 1961 ; Fine \& Vermooton, 1960). Involvement of the ureters by carcinoma of the cervix is well known, but in a recent survey no cases of rupture of the renal pelvis were described (Mallik, 1962).

It seems likely that the primary tumour in our patient caused early obstruction of the ureter, with resultant pyelitis which led to spontaneous rupture of the pelvis of the kidney, the basic lesion not being apparent at the first admission. Mixed mesodermal tumour of the uterus is rare (Pickles, 1957) and usually presents with post-menopausal bleeding or foul vaginal discharge. Our patient had no such symptoms on her first admission and a mass in the pelvis could not be felt until her second admission to hospital suggesting that the tumour was growing rapidly. This would be in keeping with the known bad prognosis of these tumours.

\section{Summary}

A case of spontaneous rupture of the kidney is described where the obstructing lesion was at the lower end of the ureter and was due to secondary spread from the rare uterine tumour, mixed mesodermal sarcoma. This case is of interest as both conditions are rare and we have been unable to find any report of a case in which the kidney has ruptured as a result of lower ureteric obstruction.

\section{Acknowledgment}

We would like to thank Mr G. E. Moloney for permission to publish details of this patient who was admitted under his care, and for his help in the preparation of this paper.

\section{References}

FERGUSSON, J.D. (1944) Ureteral stricture with perinephric urinary extravasation caused by metastases from a silent carcinoma of the stomach. Brit. J. Surg. 31, 283.

Fine, M.G. \& Vermooten, V. (1960) Spontaneous extravasation associated with excretory urography. J. Urol. 84, 409.

JEPPESEN, F.B. (1961) Spontaneous rupture of the kidney. J. Urol. 86, 489.

Mallik, M.K. Basu (1962) A study of the renal tract in cases of recurrence of carcinoma of the cervix, with special reference to its diagnostic value. Brit. J. Urol. 34, 181.

MATHE, C.P. (1932) Spontaneous rupture of the kidney. Urol. Cutan. Rev. 36, 605.

Pickles, B.G. (1957) Mesodermal mixed tumour of the uterus. Case report and review of the literature. J. Obstet. Gynec. Brit. Emp. 64, 700.

Shaw, R.E. (1957) Spontaneous rupture of the kidney. Brit.J. Surg. 45, 68.

\title{
Internal herniation through the transverse mesocolon complicated by volvulus
}

\author{
A rare complication of a rare hernia \\ R. P. Rosswick* \\ M.Sc.(Illinois), M.B., F.R.C.S. \\ Department of Surgery, St George's Hospital, London, S.W.1
}

Herniation into the lesser sac through a deficiency in the mesentery of the transverse colon is known as a mesocolic hernia even though usually there is no true peritoneal sac. The condition was first described by Pringle in 1919, and Menegaux (1934) in a classical review of the subject reviewed

\footnotetext{
*Present address: Royal Hampshire County Hospital, Winchester, Hants.
}

fifty-one cases, only five of them having a peritoneal sac. More recent reviews by Smith (1945), Gallagher (1949) and Stewart (1962) have added single cases. The purpose of this communication is to draw attention to the condition again, and to record a complication of this condition never before reported in a patient older than any other yet reported. 